\title{
Local determination of the Hubble constant and the deceleration parameter
}

\author{
David Camarena $\odot^{1}$ and Valerio Marra $\odot^{2}$ \\ ${ }^{1}$ PPGCosmo, Universidade Federal do Espírito Santo, 29075-910 Vitória, Espírito Santo, Brazil \\ ${ }^{2}$ Núcleo Cosmo-ufes and Departamento de Física, Universidade Federal do Espírito Santo, 29075-910 Vitória, Espírito Santo, Brazil
}

(Received 1 July 2019; published 9 January 2020)

\begin{abstract}
The determination of the Hubble constant $H_{0}$ from the cosmic microwave background by the Planck Collaboration (N. Aghanim et al., arXiv:1807.06209) is in tension at $4.2 \sigma$ with respect to the local determination of $H_{0}$ by the SH0ES collaboration [M. J. Reid et al., Astrophys. J. Lett. 886, L27 (2019)]. Here we improve upon the local determination, which fixes the deceleration parameter to the standard $\Lambda \mathrm{CDM}$ model value of $q_{0}=-0.55$, that is, uses information from observations beyond the local universe. First, we derive the effective calibration prior on the absolute magnitude $M_{B}$ of type Ia supernovae, which can be used in cosmological analyses in order to avoid the double counting of low-redshift supernovae. We find $M_{B}=-19.2334 \pm 0.0404$ mag. Then we use the above $M_{B}$ prior in order to obtain a determination of the local $H_{0}$ which uses only local observations and assumes only the cosmological principle, that is, large-scale homogeneity and isotropy. This is achieved by adopting an uninformative flat prior for $q_{0}$ in the cosmographic expansion of the luminosity distance. We use the latest Pantheon sample and find $H_{0}=75.35 \pm 1.68 \mathrm{~km} \mathrm{~s}^{-1} \mathrm{Mpc}^{-1}$, which features a $2.2 \%$ uncertainty, close to the $1.9 \%$ error obtained by the SH0ES Collaboration. Our determination is at the higher tension of $4.5 \sigma$ with the latest results from the Planck Collaboration that assume the $\Lambda \mathrm{CDM}$ model. Furthermore, we also constrain the deceleration parameter to $q_{0}=-1.08 \pm 0.29$, which disagrees with the Planck Collaboration at the $1.9 \sigma$ level. These estimations only use supernovae in the redshift range $0.023 \leqslant z \leqslant 0.15$.
\end{abstract}

DOI: 10.1103/PhysRevResearch.2.013028

\section{INTRODUCTION}

The $\Lambda$ CDM model, the standard model of cosmology, has been extremely successful. Assuming only general relativity and well-understood linear perturbations about a homogeneous and isotropic background model, with just six parameters it accounts for basically all cosmological observations on a vast range of scales in space and time. Its key ingredients are the cosmological constant, a constant of nature, and dark matter, yet-undetected particles which are predicted by, e.g., supersymmetric extensions of the standard model of particle physics. Although the theoretical basis for both the cosmological constant and dark matter may be rightfully questioned, the standard model has pragmatically maintained its supremacy, due to its performance and simplicity.

However, since the first release of the cosmic microwave background $(\mathrm{CMB})$ observations by the Planck Collaboration in 2013 [1], the determination of the Hubble constant $H_{0}$ based on the standard model of cosmology started to be in tension with the model-independent determination via calibrated local Supernovae Ia (SN) by the SHOES Collaboration in 2011 [2]. The initial tension of $2.4 \sigma$ worsened over the past six years. On one side, systematic uncertainties were better understood and $\mathrm{CMB}$ data accumulated $[3,4]$. On the other side, the

Published by the American Physical Society under the terms of the Creative Commons Attribution 4.0 International license. Further distribution of this work must maintain attribution to the author(s) and the published article's title, journal citation, and DOI. sample of local supernovae increased and the anchors used to calibrate them considerably improved [5-7]. The present situation is that the two determinations of the Hubble constant, one by the Planck Collaboration in 2018 [8] and the other by the SHOES Collaboration in 2019 [7], are now in tension at the considerable $4.2 \sigma$ level. (See [9] for a historical overview and [10] for the present status.)

Much work has been done trying to understand the implications of this tension: It is indeed, by far, the most severe problem the $\Lambda \mathrm{CDM}$ model is facing. On one hand, the effect of the local structure, the so-called cosmic variance on $H_{0}$, has been thoroughly studied (see [11] and references therein), as well as possible reassessments of the error budget [12-14]. On the other hand, physics beyond the standard model has been investigated, in the hope that this tension could reveal possible alternatives to the highly tuned cosmological constant and the yet-undetected dark matter (see, e.g., [15-19]). However, at the moment, it is not clear which kind of physics beyond $\Lambda \mathrm{CDM}$ could solve this crisis [20].

Here we wish to improve upon the local determination by the SHOES Collaboration, which adopts a Dirac $\delta$ prior on the deceleration parameter, centered at the standard $\Lambda \mathrm{CDM}$ model value of $q_{0}=-0.55$. In [14] it was shown that using the broad (truncated) Gaussian prior $q_{0}=-0.5 \pm 1$, it is indeed possible to obtain a competitive constraint on the Hubble constant. In what follows, first we derive the calibration prior on the absolute magnitude $M_{B}$ of supernovae Ia that was effectively used in the $H_{0}$ determination by the SHOES Collaboration. Then, using the $M_{B}$ prior and an uninformative flat prior on $q_{0}$, we obtain a competitive determination of the $H_{0}$. Indeed, our determination has an uncertainty comparable 
to the one by the SHOES Collaboration and it is at the even higher tension of $4.5 \sigma$ with the latest results from the Planck Collaboration that assume the $\Lambda \mathrm{CDM}$ model. We stress that our determination only uses local observations and only assumes the Friedmann-Lemaître-Robertson-Walker (FLRW) metric, that is, the cosmological principle, according to which the universe is homogeneous and isotropic at large scales.

This paper is organized as follows. After introducing lowredshift cosmography in Sec. II, we will obtain the effective calibration prior in Sec. III and the determination of the Hubble constant in Sec. IV using the Pantheon data set. We discuss our results in Sec. V and summarize in Sec. VI. Appendix A presents details regarding the derivation of the effective calibration prior, Appendix B reports the results relative to the Supercal supernova data set, and Appendix $\mathrm{C}$ reports the results relative to the older determination of $H_{0}$ in [6].

\section{COSMOGRAPHY}

The apparent magnitude $m_{B}^{t}$ of a supernova at redshift $z$ is given by

$$
m_{B}^{t}(z)=5 \log _{10}\left[\frac{d_{L}(z)}{1 \mathrm{Mpc}}\right]+25+M_{B},
$$

where $d_{L}(z)$ is the luminosity distance and $M_{B}$ the absolute magnitude. Using a cosmographic approach within an FLRW metric, which only assumes large-scale homogeneity and isotropy, one has

$$
d_{L}(z)=\frac{c z}{H_{0}}\left[1+\frac{\left(1-q_{0}\right) z}{2}+O\left(z^{2}\right)\right],
$$

where the Hubble constant and the deceleration parameter are defined, respectively, according to

$$
H_{0}=\left.\frac{\dot{a}(t)}{a(t)}\right|_{t_{0}}, \quad q_{0}=\left.\frac{-\ddot{a}(t)}{H(t)^{2} a(t)}\right|_{t_{0}} .
$$

Cosmography is a model-independent approach in the sense that it does not assume a specific model as it is based on the Taylor expansion of the scale factor. However, this does not mean that its parameters do not contain cosmological information. For example, the deceleration parameter is connected to the parameters of $w \mathrm{CDM}$ cosmologies according to

$$
\begin{aligned}
q_{0} & =\frac{\Omega_{\mathrm{m} 0}}{2}+\frac{1+3 w}{2} \Omega_{\mathrm{de} 0}=\frac{1+3 w \Omega_{\mathrm{de} 0}}{2} \\
& =\frac{1-3 \Omega_{\Lambda 0}}{2} \simeq-0.55
\end{aligned}
$$

where the second equality in the first line assumes spatial flatness $\left(\Omega_{\mathrm{m} 0}+\Omega_{\mathrm{de} 0}=1\right)$, the first equality in the second line assumes the $\Lambda$ CDM model $(w=-1)$, and the second one in the second line assumes the concordance value $\Omega_{\Lambda 0} \simeq 0.7$.

Combining now Eqs. (1) and (2), one has

$$
\begin{aligned}
m_{B}^{t}= & 5 \log _{10}\left[1+\frac{\left(1-q_{0}\right) z}{2}+O\left(z^{2}\right)\right] \\
& +5 \log _{10} \frac{c z H_{0}^{-1}}{1 \mathrm{Mpc}}+25+M_{B} .
\end{aligned}
$$

We are neglecting the second-order correction that contains the jerk parameter $j_{0}=\left.\frac{\dddot{a}}{H^{3} a}\right|_{t_{0}}$ because we consider only low-

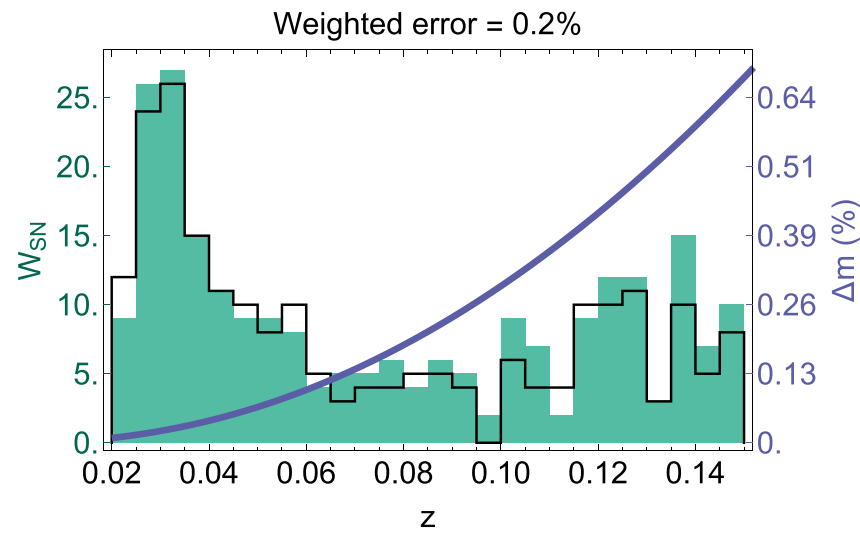

FIG. 1. Shown with a solid line is the percentage difference between the distance modulus with and without the second-order correction in the expansion of the luminosity distance of Eq. (2). The difference is always smaller than $0.7 \%$. The histogram shows the distribution $W_{S N}$ of the supernovae in the range $0.023 \leqslant z \leqslant$ 0.15 that are used for local determinations of $H_{0}$. The filled green histogram corresponds to the Pantheon sample (237 supernovae) [25] and the empty black one to the Supercal sample (217 supernovae) [26]. Using these distributions, the weighted error from neglecting the second-order correction is only $0.2 \%$.

redshift supernovae. Indeed, the latest local determinations of $H_{0}$ use supernovae in the range $0.023 \leqslant z \leqslant 0.15$, where the minimum redshift is large enough in order to reduce the impact of cosmic variance [11,21] and the maximum redshift is small enough in order to reduce the impact of cosmology in the determination of $H_{0}$. We have to bear in mind that the cosmographic expansion could be problematic if extended to high redshifts $(z>1)$. Indeed, it fails to converge [22] and also its accuracy depends on the order adopted [23]. Here we avoid these possible issues as the maximum redshift is $z=0.15$ [24]. Figure 1 shows the percentage difference with respect to the computation that considers the second-order correction, together with the distribution of the supernovae Ia that are used to determine $H_{0}$. The weighted error is $0.2 \%$, which is negligible compared to the error budget to be discussed below. Furthermore, the fact that it is safe to neglect the second-order correction implies that this analysis is valid also for spatially curved models.

\section{EFFECTIVE CALIBRATION PRIOR}

The determination of $H_{0}$ by the SH0ES Collaboration is a two-step process. On one hand, the anchors, Cepheid hosts, and calibrators are combined to produce a constraint on $M_{B}$. This step depends on the astrophysical properties of the sources and is independent of cosmology. On the other hand, the Hubble-Flow supernovae of the Supercal sample [26] are used to probe the luminosity-distance-redshift relation in order to determine $H_{0}$. In this section, using two different methods, we determine the calibration prior $f\left(M_{B}\right)$ which was effectively used in the analysis in [6].

\section{A. Demarginalization}

Within a Bayesian framework, the determination of $H_{0}$ by the SHOES Collaboration can be effectively summarized 
according to the analysis

$$
\begin{gathered}
f\left(H_{0}, M_{B} \mid \mathrm{SN}\right)=\frac{f\left(H_{0}\right) f\left(M_{B}\right) \mathcal{L}\left(\mathrm{SN} \mid H_{0}, q_{0}, M_{B}\right)}{\mathcal{E}}, \\
f\left(H_{0} \mid \mathrm{SN}\right)=\int d M_{B} f\left(H_{0}, M_{B} \mid \mathrm{SN}\right),
\end{gathered}
$$

where the posterior on $H_{0}$ was obtained by marginalizing over $M_{B}$. In the equations above, $f\left(H_{0}\right)$ is an improper flat prior on $H_{0}, \mathcal{L}$ is the likelihood, and $\mathcal{E}$ is the evidence. Here SN stands for Supercal supernovae in the range $0.023 \leqslant z \leqslant 0.15$ [26]. In addition, $f\left(M_{B}\right)$ is the informative prior on the supernova absolute magnitude and is the result of the complicated calibration of the local supernovae via the cosmic distance ladder (see [24] for details).

The likelihood is given by

$$
\mathcal{L}\left(\mathrm{SN} \mid H_{0}, q_{0}, M_{B}\right)=|2 \pi \Sigma|^{-1 / 2} e^{-\frac{1}{2} \chi^{2}\left(H_{0}, q_{0}, M_{B}\right)},
$$

where the $\chi^{2}$ function is

$$
\chi^{2}=\left\{m_{B, i}-m_{B}^{t}\left(z_{i}\right)\right\} \Sigma_{i j}^{-1}\left\{m_{B, j}-m_{B}^{t}\left(z_{j}\right)\right\},
$$

where $\Sigma$ is the supernova covariance matrix and $m_{B, i}$ are the observed apparent magnitudes at the redshifts $z_{i}$. Following the methodology of [24], we fix the nuisance parameters that control stretch and color corrections to $\alpha=0.14$ and $\beta=3.1$, correct the apparent supernova magnitudes with hosts above and below $\log _{10} M_{\text {stellar }} \sim 10$ by 0.03 mag fainter and brighter, respectively, and include an intrinsic dispersion of $\sigma_{\text {int }}=0.1$ mag together with a peculiar velocity error of $250 \mathrm{~km} / \mathrm{s}$.

The analysis of [7] fixes $q_{0}=-0.55$ in Eq. (6) and obtains

$$
H_{0}^{\operatorname{Re} 19}=73.5 \pm 1.4 \mathrm{~km} \mathrm{~s}^{-1} \mathrm{Mpc}^{-1} \text {. }
$$

Reference [6] states that the sensitivity of $H_{0}$ to knowledge of $q_{0}$ is very low as the mean $\mathrm{SN}$ redshift is only 0.07 . However, $q_{0}=-0.55$ is justified only if one uses information beyond the local universe that can constrain the properties of dark matter and dark energy [see Eq. (4)], such as data that include supernovae at $z>0.15[25,27]$. This was necessary as there were not enough local supernovae and/or their calibration was less precise so it was not possible to constrain well $H_{0}$ if $q_{0}$ was not fixed. However, as we will show below (see also [14]), the latest supernova catalog and anchors allow us to perform an analysis which is only based in the $z \leqslant 0.15$ universe. It is important to stress that the actual analysis of [6] is more complicated than the one of Eq. (6) as it involves many intermediate steps.

Here, in order to get the calibration prior $f\left(M_{B}\right)$ we solve the integral equation obtained by demanding that Eq. (7) gives the constraint of Eq. (10). Assuming a Gaussian distribution for $M_{B}$ with mean $\bar{M}_{B}$ and dispersion $\sigma_{M}$, which is also justified a posteriori by the fact that $M_{B}$ is tightly constrained by data, it is possible to marginalize analytically the twodimensional (2D) posterior in Eq. (7). The result is that $H_{0}$ is distributed according to a log-normal distribution with the parameters

$$
\begin{gathered}
\mu_{\ln }^{\mathrm{dm}}=\frac{\ln 10}{5}\left[\bar{M}_{B}+\frac{\ln 10}{5}\left(\sigma_{M}^{2}+\frac{1}{S_{0}}\right)-\frac{S_{1}}{S_{0}}\right], \\
\sigma_{\ln }^{\mathrm{dm}}=\frac{\ln 10}{5} \sqrt{\sigma_{M}^{2}+\frac{1}{S_{0}}} .
\end{gathered}
$$

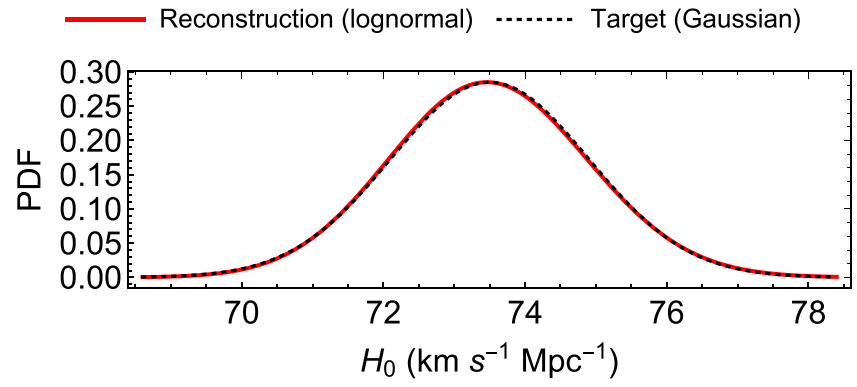

FIG. 2. Reconstruction of the determination by Reid et al. [7] of Eq. (10) using the calibration prior of Eq. (16). Also shown is a Gaussian probability distribution function (PDF) with same mean and dispersion. It is evident that the deviation from Gaussianity is negligible.

One can then match first and second moments of the lognormal distribution with Eq. (10) so that one has

$$
\mu_{\mathrm{ln}}^{\mathrm{Re} 19} \simeq 4.2971, \quad \sigma_{\ln }^{\mathrm{Re} 19} \simeq 0.019046,
$$

where we used Eqs. (A8) and (A9). The calibration prior is then given by

$$
\begin{gathered}
M_{B}^{\mathrm{dm}}=\frac{5}{\ln 10} \mu_{\ln }^{\mathrm{Re} 19}+\frac{S_{1}}{S_{0}}-\frac{\ln 10}{5}\left(\sigma_{M}^{2}+\frac{1}{S_{0}}\right), \\
\sigma_{M_{B}^{\mathrm{dm}}}^{2}=\frac{25}{\ln ^{2} 10} \sigma_{\ln }^{2 \operatorname{Re} 19}-\frac{1}{S_{0}} .
\end{gathered}
$$

The quantities $S_{0}$ and $S_{1}$ are defined in Appendix A, where the full derivation can be found. Using the previous equations, one obtains the calibration prior $f\left(M_{B}\right)$ :

$$
M_{B}^{\mathrm{dm}}=-19.2334 \pm 0.0404 \mathrm{mag} .
$$

In the computation above we considered the second-order correction in $d_{L} / z$ and fixed $j_{0}=1$ because [6] does so [see Eq. (19)]. The second-order correction shifts the prior by a small $\sim 0.1 \sigma$. The log-normal distribution on $H_{0}$ is very close to a Gaussian as shown in Fig. 2, where the log-normal $f\left(H_{0} \mid \mathrm{SN}\right)$ is compared with a Gaussian, both with mean and dispersion as in Eq. (10).

\section{B. Deconvolution}

One may obtain the calibration prior also via the following approach. The Hubble constant and the supernova absolute magnitude are connected through the equation [24]

$$
\log _{10} H_{0}\left(q_{0}, j_{0}\right)=\frac{M_{B}+5 a_{B}\left(q_{0}, j_{0}\right)+25}{5},
$$

where the "intercept" $a_{B}$ is obtained via the Hubble-Flow supernovae,

$$
\begin{gathered}
a_{B}\left(z, m_{B}, q_{0}, j_{0}\right)=\log _{10}\left[c z f\left(z, q_{0}, j_{0}\right)\right]-\frac{1}{5} m_{B}, \\
f=1+\frac{1-q_{0}}{2} z-\frac{1-q_{0}-3 q_{0}^{2}+j_{0}}{6} z^{2}+O\left(z^{3}\right),
\end{gathered}
$$

where $z$ and $m_{B}$ are the observed redshift and apparent magnitude of the supernovae. In (17) the absolute magnitude $M_{B}$ and the intercept $a_{B}$ are independent as the former is obtained via the astrophysical observations of anchors, Cepheid hosts, 
and calibrators, while the latter is obtained via Hubble-Flow supernovae that probe the cosmological luminosity-distanceredshift relation. In particular, fixing $q_{0}=-0.55$ and $j_{0}=1$ affects $a_{B}$ but not $M_{B}$. This shows clearly that it is possible to derive the constraint on $M_{B}$ by "subtracting" (or deconvolving) the contribution of $a_{B}$ from the constraint on $H_{0}$.

The constraint on $a_{B}$ using the Supercal sample [26] is [24]

$$
a_{B}\left(q_{0}=-0.55, j_{0}=1\right)=0.71273 \pm 0.00176,
$$

which can be obtained via [28]

$$
a_{B}=\frac{\sum_{i, j}\left(C^{-1}\right)_{i j} a_{B, i}}{\sum_{i, j}\left(C^{-1}\right)_{i j}}, \quad \sigma_{a_{B}}^{2}=\frac{1}{\sum_{i, j}\left(C^{-1}\right)_{i j}},
$$

where $a_{B, i}=a_{B}\left(z_{i}, m_{B, i}, q_{0}, j_{0}\right)$ are the intercept measurements with covariance matrix $C$. After propagating the errors on $z$ (mostly peculiar velocity) on $m_{B}$, it is $C=\Sigma / 25$, where $\Sigma$ is the covariance matrix for $m_{B}$. Regarding $m_{B}$ and $\Sigma$, we follow the methodology of [24] as described in the preceding section.

One can then proceed in two ways. The simplest approach is just to perform first-order error propagation

$$
\begin{aligned}
M_{B}^{\mathrm{e}} & =5\left(\log _{10} H_{0}^{\mathrm{Re} 19}-a_{B}-5\right)=-19.2322, \\
\sigma_{M}^{\mathrm{e}} & =\sqrt{\left(\frac{5}{\ln 10} \frac{\sigma_{H_{0}^{\mathrm{Re}} 19}}{H_{0}^{\mathrm{Re} 19}}\right)^{2}-25 \sigma_{a_{B}}^{2}}=0.0404 .
\end{aligned}
$$

Alternatively, one can make a change of variable so that the higher moments are included. Adopting Gaussian distributions for $M_{B}$ and $a_{B}$, it follows that $H_{0}$ is distributed according to the log-normal distribution of Eq. (A5) with the parameters

$$
\begin{gathered}
\mu_{\ln }^{\mathrm{dc}}=\frac{\ln 10}{5}\left(\bar{M}_{B}+5 a_{B}+25\right), \\
\sigma_{\ln }^{\mathrm{dc}}=\frac{\ln 10}{5} \sqrt{\sigma_{M}^{2}+25 \sigma_{a_{B}}^{2}},
\end{gathered}
$$

so the calibration prior is given by

$$
\begin{gathered}
M_{B}^{\mathrm{dc}}=M_{B}^{\mathrm{dm}}+\frac{\ln 10}{5}\left(\sigma_{M}^{2}+\frac{1}{S_{0}}\right), \\
\sigma_{M_{B}^{\mathrm{dc}}}^{2}=\sigma_{M_{B}^{\mathrm{dm}}}^{2},
\end{gathered}
$$

where we used the fact that

$$
5 a_{B}+25=-\frac{S_{1}}{S_{0}}, \quad 25 \sigma_{a_{B}}^{2}=\frac{1}{S_{0}} .
$$

Using the previous equations, one obtains the calibration prior $f\left(M_{B}\right)$,

$$
M_{B}^{\mathrm{dc}}=-19.2326 \pm 0.0404 \mathrm{mag},
$$

which confirms the validity of the first-order estimate of Eq. (22). We see that the demarginalization and deconvolution approaches give very similar results: The variance of the prior is actually identical while the central value differs by a negligible amount:

$$
M_{B}^{\mathrm{dc}}-M_{B}^{\mathrm{dm}} \simeq \frac{5}{\ln 10}\left(\frac{\sigma_{H_{0}^{\mathrm{Re} 19}}}{H_{0}^{\mathrm{Re} 19}}\right)^{2} \simeq 8 \times 10^{-4} .
$$

We will adopt the demarginalization result as the analysis of the next section generalizes its methodology.

\section{Robustness}

The computation of the calibration prior with the methods of the previous sections is possible because its determination is independent of the cosmological Hubble flow that is used to determine $H_{0}$. In particular, $M_{B}$ is independent of the value adopted for the cosmographic parameters $q_{0}$ and $j_{0}$ as their effect is to alter the luminosity distance and not $M_{B}$. This would not be the case if SHOES constrained Hubble flow and $M_{B}$ together as, in this case, $M_{B}$ would be correlated with the Hubble-flow supernovae.

Even though the methodology is well posed, there could be astrophysical or cosmological effects that could bias the determination of $M_{B}$. A calibration bias could be produced by the coherent flow of the local supernovae, akin to cosmic variance [11]. However, both the determination of Eq. (10) and the Supercal data set have already been corrected for such effects using velocity fields derived from galaxy density fields [26]. Another bias could be due to correlations between supernovae and environmental properties such as star formation rate and metallicity. Reference [6] concluded that these correlations are not significant, so they should not impact Eq. (16) either (see, however, [29,30]). Therefore, we conclude that our determination of the $M_{B}$ prior is robust and correctly encodes the supernova calibration via the distance ladder.

\section{Calibration prior in cosmological analyses}

The calibration prior on $M_{B}$ of Eq. (16) can be meaningfully used in cosmological analyses instead of the corresponding $H_{0}$ determination. Indeed, there are 175 supernovae in common between the Supercal and Pantheon data sets in the range $0.023 \leqslant z \leqslant 0.15$ and, in the standard analysis, these supernovae are used twice: once for the $H_{0}$ determination and once when constraining the cosmological parameters. This induces a covariance between $H_{0}$ and the other parameters which could bias cosmological inference. The advantage of adopting the calibration prior is that these 175 supernovae are used only once, avoiding any potential bias.

\section{DETERMINATION OF LOCAL $H_{0}$}

Here we wish to obtain a determination of $H_{0}$ that is based solely on the local universe $(z \leqslant 0.15)$ and that is truly independent of any cosmological assumptions besides the cosmological principle and the astrophysics of anchors, Cepheid hosts, and calibrators. We achieve the latter by considering an uninformative flat prior on $q_{0}$ and marginalizing the posterior also over $q_{0}$, that is,

$$
\begin{aligned}
& f\left(H_{0}, q_{0}, M_{B} \mid \mathrm{SN}\right)=\frac{f\left(H_{0}\right) f\left(q_{0}\right) f\left(M_{B}\right) \mathcal{L}}{\mathcal{E}}, \\
& f\left(H_{0} \mid \mathrm{SN}\right)=\int d M_{B} d q_{0} f\left(H_{0}, q_{0}, M_{B} \mid \mathrm{SN}\right),
\end{aligned}
$$

where $f\left(q_{0}\right)$ is the flat ignorance prior on $q_{0}$. The method of [6] is recovered if we set $f\left(q_{0}\right)=\delta\left(q_{0}+0.55\right)$. In this analysis we adopt the more recent Pantheon supernova sample [25], which features 237 supernovae in the redshift range $0.023 \leqslant z \leqslant 0.15$ (see Fig. 1). In this case the covariance matrix and the apparent supernova magnitudes do not depend 


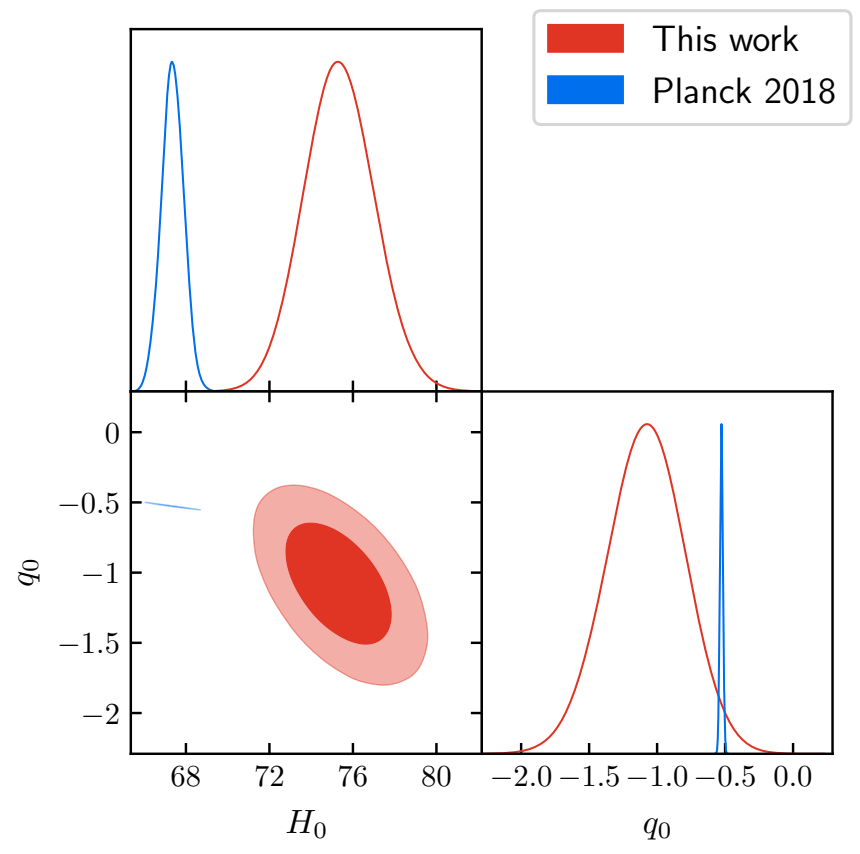

FIG. 3. Determination of the Hubble constant $H_{0}$ and the deceleration parameter $q_{0}$ from the Pantheon supernovae in the redshift range $0.023 \leqslant z \leqslant 0.15$ [25]. The constraints have been marginalized over the absolute magnitude $M_{B}$. Also shown are the marginalized 2D constraints on $H_{0}$ and $q_{0}$ from the CMB-only Planck Collaboration analysis that assumes the standard flat $\Lambda \mathrm{CDM}$ model [8]. The tension between the two determinations is at the $4.5 \sigma$ level.

on the nuisance parameters that control stretch and color corrections, and the correction for the apparent magnitudes with hosts above and below $\log _{10} M_{\text {stellar }} \sim 10$ is already applied. Also, the covariance matrix includes all the uncertainties. The results relative to the Supercal sample are given in Appendix B.

We have used EMCEE [31], an open-source sampler for Markov chain Monte Carlo (MCMC), to sample the posterior and GETDIST [32] for analyzing the chains. The result of this analysis is presented in Fig. 3. We obtain

$$
\begin{gathered}
H_{0}^{\text {loc }}=75.35 \pm 1.68 \mathrm{~km} \mathrm{~s}^{-1} \mathrm{Mpc}^{-1}, \\
q_{0}^{\text {loc }}=-1.08 \pm 0.29 .
\end{gathered}
$$

The latest CMB-only constraint from the Planck Collaboration (see Table 2 in [8]) is

$$
H_{0}^{\text {Planck }}=67.36 \pm 0.54 \mathrm{~km} \mathrm{~s}^{-1} \mathrm{Mpc}^{-1}
$$

and the tension with our determination now reaches $4.5 \sigma$ :

$$
\frac{\left|H_{0}^{\text {loc }}-H_{0}^{\text {Planck }}\right|}{\sqrt{\sigma_{H_{0}}^{2}+\sigma_{H_{0}^{\text {Planck }}}^{2}}} \simeq 4.52 .
$$

As it is clear from Fig. 3, low-redshift supernovae are able to constrain not only $H_{0}$ but also $q_{0}$, whose distribution is peaked at values lower than the standard model one of $q_{0}=-0.55$. However, the deceleration parameter is not yet tightly constrained and the tension between our determination and the one by Planck is at the $1.9 \sigma$ level. Note that the $\Lambda \mathrm{CDM}$ model cannot give values of $q_{0}$ below -1 .
Finally, it is interesting to compare directly the marginalized 2D posterior on $H_{0}$ and $q_{0}$ from CMB and low- $z$ supernova observations. We obtained the CMB posterior using the MCMC chain from the CMB-only Planck analysis available from [33]. The tension in the $H_{0}-q_{0}$ plane is also presented in Fig. 3.

In order to quantity the tension we adopt the index of inconsistency (IOI) [34], which directly generalizes the estimator of Eq. (35). We find

$$
\begin{aligned}
\sqrt{2 \mathrm{IOI}} & \equiv \sqrt{\delta^{T}\left(C_{\text {loc }}+C_{\text {Planck }}\right)^{-1} \delta} \simeq 4.54, \\
\delta & =\left\{H_{0}^{\text {loc }}-H_{0}^{\text {Planck }}, q_{0}^{\text {loc }}-q_{0}^{\text {Planck }}\right\},
\end{aligned}
$$

where $C$ are the covariance matrices on $H_{0}$ and $q_{0}$ from the analysis of Fig. 3 and $\delta$ is the difference vector. Note that these estimators assume Gaussianity and that the posteriors on $H_{0}$ and $q_{0}$ are very close to Gaussian. The tension in the $H_{0}-q_{0}$ plane is again very strong: $4.5 \sigma$.

\section{DISCUSSION}

Our determination of the Hubble constant of Eq. (32) is based on the calibration prior of Eq. (16) and on the lowredshift cosmographic expansion of Eq. (2). While the validity of the calibration prior depends on the standardizable nature of supernovae Ia, a process that includes corrections due to color, stretch, and host-galaxy mass, the validity of the cosmographic analysis is solely based on the approximation that the FLRW metric provides a good description of our universe at large scales. While it is possible to test directly this hypothesis [35-38], the validity of the FLRW metric is a direct consequence of assuming the cosmological principle, according to which the universe is homogeneous and isotropic at large scales. Therefore, as far as cosmological assumptions are concerned, the determination of Eq. (32) only assumes the cosmological principle.

While our goal here was to improve the local determination of $H_{0}$ by considering a flat prior on $q_{0}$, it is also possible to perform a more thorough reanalysis of the cosmic distance ladder and consider the impact of non-Gaussianities [14], hyperparameters [12], and a blinded pipeline [13]. In particular, our results are in agreement with the findings of [14], which, using a Bayesian hierarchical model, also found that data prefer a lower value of $q_{0} \approx-1$ and so, owing to their anticorrelation, a higher value of $H_{0}$. In particular, Ref. [14] adopted a broad (truncated) Gaussian prior $q_{0}=-0.5 \pm 1$, similar to our improper flat prior, and obtained a value of $H_{0}$ which is $\sim 1 \mathrm{~km} \mathrm{~s}^{-1} \mathrm{Mpc}^{-1}$ higher than their baseline $H_{0}$ value. This is in good agreement with our results relative to the Supercal sample, the one adopted in [14]. Indeed, the determination of Eq. (B1) is $\sim 1 \mathrm{~km} \mathrm{~s}^{-1} \mathrm{Mpc}^{-1}$ rather than the $H_{0}^{\text {Re19 }}$ of Eq. (10).

It is also possible to extend the cosmic ladder to baryon acoustic oscillation (BAO) and CMB observations. Reference [39] constrained $H_{0}$ via a cosmographic inverse ladder approach, using BAO measurements to break the degeneracy between $M_{B}$ and $H_{0}$ and finding a value of the Hubble constant very close to the Planck one: $H_{0}^{\mathrm{M} 18}=$ $67.8 \pm 1.3 \mathrm{~km} \mathrm{~s}^{-1} \mathrm{Mpc}^{-1}$. The cosmographic inverse ladder approach propagates the prior on the sound horizon from 
CMB to the BAO scale. However, the calibration of $M_{B}$ depends on the luminosity-distance-redshift relation of supernovae both at low and high redshift. This introduces correlations between $M_{B}$ and the properties of dark energy, which can explain why our cosmology-independent determination of $q_{0}$ differs from the constraint $q_{0}^{\mathrm{M} 18}=-0.37 \pm 0.15$ obtained in [39]. Finally, a low local value of $q_{0} \approx-1$ was also obtained in [40], where the cosmic ladder was extended to BAO and CMB observations using the distance-duality relation instead of a high-redshift cosmographic expansion.

\section{CONCLUSION}

We obtained the effective local calibration prior on the absolute magnitude $M_{B}$ of Supernovae Ia, which we used to obtain a determination of the Hubble constant from the local universe that assumes only large-scale homogeneity and isotropy: $H_{0}=75.35 \pm 1.68 \mathrm{~km} \mathrm{~s}^{-1} \mathrm{Mpc}^{-1}$. Our determination uses the latest Pantheon sample. As shown in Fig. 3, the allowed values are in stronger tension with what the CMB predicts if the standard model of cosmology is valid. The $H_{0}$ crisis is approaching the $5 \sigma$ level. We stress that our determination relies only on supernovae in the redshift range $0.023 \leqslant z \leqslant 0.15$. If, on one hand, this limits the available information as there are many more supernovae at higher redshifts, on the other hand, this restricted redshift range makes this analysis local. We would like to stress that the calibration prior on $M_{B}=-19.2334 \pm 0.0404$ mag can be meaningfully used in cosmological analyses, instead of the corresponding $H_{0}$ determination, in order to avoid the double counting of low-redshift supernovae.

The fact that both Hubble constant and deceleration parameter differ seems to suggest that a new phenomenon is affecting both the sound horizon and the recent expansion rate of the universe. Early dark energy may be a promising candidate [16]. The increased statistical significance of our determination of $H_{0}$ reinforces this suggestion.

\section{ACKNOWLEDGMENTS}

D.C. thanks CAPES for financial support. V.M. thanks CNPq and FAPES for partial financial support. We thank the referee for suggesting the alternative method used in Sec. III B.

\section{APPENDIX A: DERIVATION OF THE DEMARGINALIZATION}

Here we explain how we obtained Eqs. (11) and (12). First, we define

$$
\tilde{m}_{B}^{t} \equiv m_{B}^{t}-M_{B}+\operatorname{logh}
$$

where we used the shorthand notation $\operatorname{logh}=5 \log _{10} H_{0}$. We can then rewrite Eq. (9) as

$$
\begin{aligned}
\chi^{2} & =\left\{W_{i}+\operatorname{logh}-M_{B}\right\} \Sigma_{i j}^{-1}\left\{W_{i}+\operatorname{logh}-M_{B}\right\} \\
& =S_{0}\left(M_{B}-\operatorname{logh}-S_{1} / S_{0}\right)^{2}+\mathrm{const},
\end{aligned}
$$

where 'const' consists of all terms that do not depend on $H_{0}$ and $M_{B}$ and we defined

$$
\begin{aligned}
& W_{i}=m_{B, i}-\tilde{m}_{B}^{t}\left(z_{i}\right), \quad V_{i}=1, \\
& S_{0}=V \cdot \Sigma^{-1} \cdot V^{T}, \\
& S_{1}=W \cdot \Sigma^{-1} \cdot V^{T} .
\end{aligned}
$$

The unnormalized posterior of Eq. (6) is then

$$
f\left(H_{0}, M_{B} \mid \mathrm{SN}\right) \propto e^{-\frac{\left(M-\bar{M}_{B}\right)^{2}}{2 \sigma_{M}^{2}}} e^{-\frac{1}{2} \chi^{2}\left(H_{0}, M_{B}\right)},
$$

where an improper flat prior on $H_{0}$ was adopted. Equation (A4) can then be integrated over $M_{B}$ so that the normalized posterior on $H_{0}$ is distributed according to the log-normal distribution

$$
\begin{aligned}
f\left(H_{0} \mid \mathrm{SN}\right) & =\int d M_{B} f\left(H_{0}, M_{B} \mid \mathrm{SN}\right) \\
& =\frac{1}{H_{0} \sqrt{2 \pi} \sigma_{\ln }} \exp -\frac{\left(\ln H_{0}-\mu_{\ln }\right)^{2}}{2 \sigma_{\ln }^{2}},
\end{aligned}
$$

with the parameters

$$
\begin{gathered}
\mu_{\ln }^{\mathrm{dm}}=\frac{\ln 10}{5}\left[\bar{M}_{B}+\frac{\ln 10}{5}\left(\sigma_{M}^{2}+\frac{1}{S_{0}}\right)-\frac{S_{1}}{S_{0}}\right], \\
\sigma_{\ln }^{\mathrm{dm}}=\frac{\ln 10}{5} \sqrt{\sigma_{M}^{2}+\frac{1}{S_{0}}} .
\end{gathered}
$$

Finally, the mean and variance of the log-normal distribution are

$$
\begin{gathered}
\bar{H}_{0}=e^{\mu_{\ln }+\frac{1}{2} \sigma_{\ln }^{2}}, \\
\sigma_{H_{0}}^{2}=\left(e^{\sigma_{\ln }^{2}}-1\right) e^{2 \mu_{\ln }+\sigma_{\ln }^{2}} .
\end{gathered}
$$

\section{APPENDIX B: ANALYSIS WITH THE SUPERCAL SAMPLE}

Here we present the results using the Supercal supernova catalog [26] that was used in [6,24]. It features 217 supernovae in the redshift range $0.023 \leqslant z \leqslant 0.15$ (see Fig. 1). Figure 4 shows the results of this analysis, which are compared with the ones of Sec. IV that use the more recent Pantheon sample. We obtain

$$
\begin{gathered}
H_{0}^{\mathrm{loc}}=74.62 \pm 1.54 \mathrm{~km} \mathrm{~s}^{-1} \mathrm{Mpc}^{-1}, \\
q_{0}^{\mathrm{loc}}=-0.90 \pm 0.22,
\end{gathered}
$$

which are in tension with the determinations of $H_{0}$ and $q_{0}$ by the Planck Collaboration at the $4.5 \sigma$ and $1.7 \sigma$ levels, respectively. The tension in the $H_{0}-q_{0}$ plane is $4.5 \sigma$. There is good agreement between the results that use the Supercal and Pantheon data sets.

The previous results were obtained using the methodology of [24], that is, fixing the nuisance parameters that control stretch and color corrections to $\alpha=0.14$ and $\beta=3.1$, correcting the apparent supernova magnitudes with hosts above and below $\log _{10} M_{\text {stellar }} \sim 10$ by 0.03 mag fainter and brighter, respectively, and including an intrinsic dispersion of $\sigma_{\text {int }}=$ $0.1 \mathrm{mag}$ together with a peculiar velocity error of $250 \mathrm{~km} / \mathrm{s}$. 


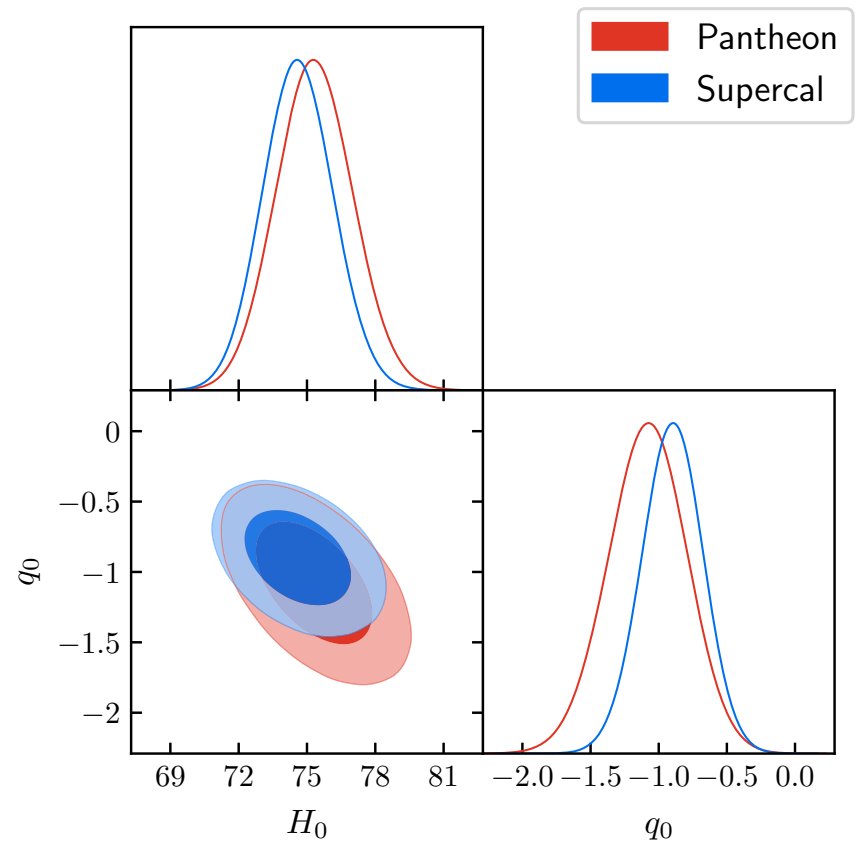

FIG. 4. Comparison between the analysis that uses the Supercal supernova sample and the one of Fig. 3 that uses the Pantheon sample. The agreement is good.
If instead we adopt a flat prior on $\alpha$ and $\beta$ we obtain basically the same results:

$$
\begin{gathered}
H_{0}^{\mathrm{loc}}=74.85 \pm 1.57 \mathrm{~km} \mathrm{~s}^{-1} \mathrm{Mpc}^{-1}, \\
q_{0}^{\mathrm{loc}}=-0.98 \pm 0.24
\end{gathered}
$$

\section{APPENDIX C: ANALYSIS RELATIVE TO THAT OF RIESS et al.}

Here we report the results relative to the measurement by Riess et al. in [6]:

$$
H_{0}^{\mathrm{R} 19}=74.03 \pm 1.42 \mathrm{~km} \mathrm{~s}^{-1} \mathrm{Mpc}^{-1} \text {. }
$$

The calibration prior is

$$
M_{B}^{\mathrm{dm}}=-19.2178 \pm 0.0407 \mathrm{mag},
$$

which gives the determinations

$$
\begin{gathered}
H_{0}^{\mathrm{loc}}=75.89 \pm 1.70 \mathrm{~km} \mathrm{~s}^{-1} \mathrm{Mpc}^{-1}, \\
q_{0}^{\mathrm{loc}}=-1.08 \pm 0.29,
\end{gathered}
$$

which are in tension with the corresponding CMB-only constraints from the Planck Collaboration at the $4.8 \sigma$ and $1.9 \sigma$ levels, respectively. The tension in the $H_{0}-q_{0}$ plane is at the $4.8 \sigma$ level.
[1] P. A. R. Ade et al. (Planck Collaboration), Planck 2013 results. XVI. Cosmological parameters, Astron. Astrophys. 571, A16 (2014).

[2] A. G. Riess, L. Macri, S. Casertano, H. Lampeitl, H. C. Ferguson et al., A 3 space telescope and wide field camera 3, Astrophys. J. 730, 119 (2011).

[3] D. N. Spergel, R. Flauger, and R. Hložek, Planck data reconsidered, Phys. Rev. D 91, 023518 (2015).

[4] N. Aghanim et al. (Planck Collaboration), Planck intermediate results. XLVI. Reduction of large-scale systematic effects in HFI polarization maps and estimation of the reionization optical depth, Astron. Astrophys. 596, A107 (2016).

[5] G. Pietrzyński, D. Graczyk, A. Gallenne, W. Gieren, I. B. Thompson, B. Pilecki, P. Karczmarek, M. Górski, K. Suchomska, M. Taormina et al., A distance to the Large Magellanic Cloud that is precise to one per cent, Nature (London) 567, 200 (2019).

[6] A. G. Riess, S. Casertano, W. Yuan, L. M. Macri, and D. Scolnic, Large Magellanic Cloud Cepheid standards provide a $1 \%$ foundation for the determination of the Hubble constant and stronger evidence for physics beyond LambdaCDM, Astrophys. J. 876, 85 (2019).

[7] M. J. Reid, D. W. Pesce, and A. G. Riess, An improved distance to NGC 4258 and its implications for the hubble constant, Astrophys. J. Lett. 886, L27 (2019).

[8] N. Aghanim et al. (Planck Collaboration), Planck 2018 results. VI. Cosmological parameters, arXiv:1807.06209.

[9] W. L. Freedman, Cosmology at a crossroads, Nat. Astron. 1, 0121 (2017).

[10] L. Verde, T. Treu, and A. G. Riess, Tensions between the early and late Universe, Nat. Astron. 3, 891 (2019).
[11] D. Camarena and V. Marra, Impact of the cosmic variance on $H_{0}$ on cosmological analyses, Phys. Rev. D 98, 023537 (2018).

[12] W. Cardona, M. Kunz, and V. Pettorino, Determining $H_{0}$ with Bayesian hyper-parameters, J. Cosmol. Astropart. Phys. 1703 056 (2017).

[13] B. R. Zhang, M. J. Childress, T. M. Davis, N. V. Karpenka, C. Lidman, B. P. Schmidt, and M. Smith, A blinded determination of $H_{0}$ from low-redshift type Ia supernovae, calibrated by Cepheid variables, Mon. Not. R. Astron. Soc. 471, 2254 (2017).

[14] S. M. Feeney, D. J. Mortlock, and N. Dalmasso, Clarifying the Hubble constant tension with a Bayesian hierarchical model of the local distance ladder, Mon. Not. R. Astron. Soc. 476, 3861 (2018).

[15] K. Vattis, S. M. Koushiappas, and A. Loeb, Dark matter decaying in the late Universe can relieve the $H_{0}$ tension, Phys. Rev. D 99, 121302 (2019).

[16] V. Poulin, T. L. Smith, T. Karwal, and M. Kamionkowski, Early Dark Energy Can Resolve the Hubble Tension, Phys. Rev. Lett. 122, 221301 (2019).

[17] R.-Y. Guo, J.-F. Zhang, and X. Zhang, Can the $H_{0}$ tension be resolved in extensions to $\Lambda \mathrm{CDM}$ cosmology? J. Cosmol. Astropart. Phys. 1902, 054 (2019).

[18] E. Ó. Colgáin, M. H. P. M. van Putten, and H. Yavartanoo, de Sitter Swampland, $H_{0}$ tension \& observation, Phys. Lett. B 793, 126 (2019).

[19] E. Di Valentino, A. Melchiorri, and J. Silk, Reconciling Planck with the local value of $H_{0}$ in extended parameter space, Phys. Lett. B 761, 242 (2016).

[20] L. Knox and M. Millea, The Hubble hunter's guide, arXiv:1908.03663. 
[21] V. Marra, L. Amendola, I. Sawicki, and W. Valkenburg, Cosmic Variance and the Measurement of the Local Hubble Parameter, Phys. Rev. Lett. 110, 241305 (2013).

[22] C. Cattoen and M. Visser, The Hubble series: Convergence properties and redshift variables, Class. Quantum Grav. 24, 5985 (2007).

[23] M.-J. Zhang, H. Li, and J.-Q. Xia, What do we know about cosmography, Eur. Phys. J. C 77, 434 (2017).

[24] A. G. Riess et al., A $2.4 \%$ determination of the local value of the Hubble constant, Astrophys. J. 826, 56 (2016).

[25] D. M. Scolnic et al., The complete light-curve sample of spectroscopically confirmed SNe Ia from Pan-STARRS1 and cosmological constraints from the combined Pantheon sample, Astrophys. J. 859, 101 (2018).

[26] D. Scolnic et al., Supercal: Cross-calibration of multiple photometric systems to improve cosmological measurements with type Ia supernovae, Astrophys. J. 815, 117 (2015).

[27] M. Betoule et al. (SDSS Collaboration), Improved cosmological constraints from a joint analysis of the SDSS-II and SNLS supernova samples, Astron. Astrophys. 568, A22 (2014).

[28] M. Schmelling, Averaging correlated data, Phys. Scr. 51, 6769 (1995).

[29] Y.-L. Kim, M. Smith, M. Sullivan, and Y.-W. Lee, Environmental dependence of type Ia supernova luminosities from a sample without a local-global difference in host star formation, Astrophys. J. 854, 24 (2018).

[30] M. Rigault et al. (Nearby Supernova Factory Collaboration), Strong dependence of type Ia supernova standardization on the local specific star formation rate, arXiv:1806.03849.
[31] D. Foreman-Mackey, D. W. Hogg, D. Lang, and J. Goodman, emcee: The MCMC hammer, Publ. Astron. Soc. Pac. 125, 306 (2013).

[32] A. Lewis, GetDist: A Python package for analysing Monte Carlo samples, arXiv:1910.13970, available at https://getdist. readthedocs.io.

[33] esa.int/Planck.

[34] W. Lin and M. Ishak, Cosmological discordances: A new measure, marginalization effects, and application to geometry versus growth current data sets, Phys. Rev. D 96, 023532 (2017).

[35] W. Valkenburg, V. Marra, and C. Clarkson, Testing the Copernican principle by constraining spatial homogeneity, Mon. Not. R. Astron. Soc. 438, L6 (2014).

[36] M. Scrimgeour et al., The WiggleZ Dark Energy Survey: The transition to large-scale cosmic homogeneity, Mon. Not. R. Astron. Soc. 425, 116 (2012).

[37] V. Marra and D. Sapone, Null tests of the standard model using the linear model formalism, Phys. Rev. D 97, 083510 (2018).

[38] R. S. Gonçalves, G. C. Carvalho, C. A. P. Bengaly, J. C. Carvalho, and J. S. Alcaniz, Measuring the scale of cosmic homogeneity with SDSS-IV DR14 quasars, Mon. Not. R. Astron. Soc. 481, 5270 (2018)

[39] E. Macaulay et al. (DES Collaboration), First cosmological results using type Ia supernovae from the Dark Energy Survey: Measurement of the Hubble constant, Mon. Not. R. Astron. Soc. 486, 2184 (2019).

[40] D. Camarena and V. Marra, A new method to build the (inverse) distance ladder, arXiv:1910.14125. 